\title{
Generation of a de novo transcriptome from equine lamellar tissue
}

\author{
Heather M. Holl', Shan Gao ${ }^{2}$, Zhangjun Fei ${ }^{2}$, Caroline Andrews ${ }^{3}$ and Samantha A. Brooks ${ }^{*}$
}

\begin{abstract}
Background: Laminitis, the structural failure of interdigitated tissue that suspends the distal skeleton within the hoof capsule, is a devastating disease that is the second leading cause of both lameness and euthanasia in the horse. Current transcriptomic research focuses on the expression of known genes. However, as this tissue is quite unique and equine gene annotation is largely derived from computational predictions, there are likely yet uncharacterized transcripts that may be involved in the etiology of laminitis. In order to create a novel annotation resource, we performed whole transcriptome sequencing of sagittal lamellar sections from one control and two laminitis affected horses.

Results: Whole transcriptome sequencing of the three samples resulted in 113 million reads. Overall, $88 \%$ of the reads mapped to the equCab2 reference genome, allowing for the identification of 119,430 SNPs. The de novo assembly generated around 75,000 transcripts, of which 36,000 corresponded to known annotations. Annotated transcript models are hosted in a public data repository and thus can be easily accessed or loaded into genome browsers. RT-PCR of 12 selected assemblies confirmed structure and expression in lamellar tissue.
\end{abstract}

Conclusions: Transcriptome sequencing represents a powerful tool to expand on equine annotation and identify novel targets for further laminitis research.

Keywords: Equine, transcriptome, laminitis, RNA-seq, assembly

\section{Background}

Laminae are interdigitated dermal and epidermal tissues found in the hooves of livestock that form the attachment to the distal skeleton. Equids have an additional specialization in the form of secondary laminae that project from the primary laminae which further increase the surface area and thus strengthen this connection [1]. The junction between dermal and epidermal laminae must be strong enough to withstand the forces of weight bearing and motion without separation, while providing sufficient flexibility to absorb concussive forces and allow growth. Inflammation of the laminae (laminitis) is a devastating disease that can lead to separation of these tissues and a rotation of the third phalanx (P3) away from the hoof wall.

The etiology of laminitis is poorly understood. Many risk factors have been identified in the horse, including inflammation in other parts of the body, sepsis, metabolic

\footnotetext{
* Correspondence: samantha.brooks@ufl.edu

'Department of Animal Sciences, University of Florida, Gainesville, FL 32611, USA

Full list of author information is available at the end of the article
}

conditions, or mechanical stress [2]. Currently, as there are very few treatments available, prevention through avoiding known risk factors is recommended. In the early stages of laminitis (either pre-clinical symptoms or at the onset of lameness), prolonged cooling of the hooves in ice water has been shown to reduce severity of the disease and prevent separation of the laminae [3]. However, if adequate treatment is not provided promptly, euthanasia is often the result. A study from the United States Department of Agriculture in 1998 estimated the annual cost of lameness at $\$ 678$ million, with laminitis accounting for $15 \%$ of the reported cases [4]. The American Association of Equine Practitioners has specifically identified laminitis as the disease most frequently reported as needing more research [5].

Several methods have been devised to experimentally induce laminitis, including carbohydrate overload, oligofructose overload, and black walnut extract administration. Although all of these models will result in the disease, key differences in physiological response (as compared to the natural etiology) have been demonstrated [6, 7]. However, as natural cases can be much more difficult to acquire, 
these models continue to serve an important role in research.

Gene expression has been applied in studies to better understand the disease process. However, much of this research has focused on the expression of few known genes, using $\mathrm{qPCR}$ to target specific pathways [8-11]. Only two studies have attempted a transcriptome-wide view of laminitis. The first commercially available wholetranscriptome equine-specific microarray was not published until 2009, therefore early studies attempted two different approaches. The first study chose to use crossspecies hybridization with the bovine gene expression chip, identifying 155 out of the 15,000 genes assayed to be significantly up-regulated [12]. They were unable to identify any down-regulated genes, which was likely due to the high false-negative rate associated with imperfect hybridization. A second study instead generated a custom equine-specific array with 3076 targets derived from leukocyte EST libraries [13]. Less than 100 of these genes were found to have significant differential expression.

Both of these projects, and any current work utilizing microarrays, are hindered by insufficient genome annotation in the horse. The only major annotation attempt used an older sequencing technology, generating $35 \mathrm{bp}$ reads from eight diverse tissue types [14]. They identified that $48 \%$ of genes displayed tissue-specific expression patterns, with $7 \%$ of the genes only found in one tissue type. However, this data was not incorporated into automatic annotation pipelines for the popular genome browsers, and lamellar tissue was not included in sequencing. Using this data, the authors also demonstrated there were 428 genes completely lacking in equine annotation, even though many of these genes have data in other species [15].

Whole transcriptome sequencing (RNA-seq) is a promising solution for interrogation of gene structure and expression, especially in a divergent tissue like the hoof. RNA-seq is a hypothesis-free examination of all cDNA in a given sample, allowing for the identification of unique features such as unannotated transcription, splice sites, allele-specific expression, anti-sense expression, and alternative poly-adenylation [16-18]. Additionally, technical variation is reportedly low, with high reproducibility between lanes [19]. Studies have continuously demonstrated high correlation between microarray differential expression studies and RNA-seq strategies, noting the main difference is improved sensitivity for low-abundance transcripts by RNA-seq [20, 21]. However, as RNA-seq is still considerably more expensive and computationally intense than microarrays, much mainstream research still relies on microarrays or qPCR.

The objective of this study was to produce a transcriptome resource for the study of laminitis. Given that recent studies rely heavily on qPCR, the generation of a set of equine, hoof-specific transcripts can greatly benefit in the selection of novel targets for expression studies. Current annotation is largely based on computational predictions and gene models from other species, among which there is not a good physiological model for the laminae. Additionally, while there have been a few equine RNA-seq studies, raw data is often only placed in public databases and not fully processed or curated [14, 22-24]. Thus these valuable datasets are difficult to access and may require intensive bioinformatic analysis before use in subsequent projects, and sadly are often underutilized.

\section{Results}

\section{Illumina sequencing and assembly}

Whole transcriptome sequencing of the three samples in this experiment generated a total of 112,979,003 reads. Sequencing data from all three individuals was pooled for assembly in order to capture genes that may be rare or unique to the laminitic state. After filtering, 87,598,529 high-quality reads remained. The iAssembler pipeline was used to correct for misassemblies due to heterozygosity (either within or between individuals) [25]. A summary of assembly metrics can be found in Table 1 [26]. The number of unigenes (unique transcripts) mapped per locus ranged from 1 to 139 , averaging 2.44 isoforms representing 25,580 loci. Many of these unigenes are shorter transcripts covering only a single exon or splice junction, partially due to low-expression transcripts lacking sufficient coverage for assembly (Fig. 1). Considering only the longer $3+$ exon transcripts resulted in similar statistics (Table 2).

Overall, $88 \%$ of raw sequencing reads mapped to the equCab2 reference genome [27]. The GATK recommended pipeline identified a total of 131,034 SNPs [28-30]. We filtered the assembly to remove any alignments matching repeat regions, and then removed SNP calls that fell outside of our transcript models, reducing potential false positive SNPs resulting from incorrectly mapped spliced reads. The 119,430 SNPs that remained $(91.1 \%)$ were submitted to dbSNP at NCBI (Table 3).

Table 1 De novo assembly statistics

\begin{tabular}{ll}
\hline Metrics & Raw Assembly \\
\hline Total reads (100 bp) & $112,979,003$ \\
Reads after filtering & $86,275,849$ \\
Average read length after filtering & $88.3 \mathrm{bp}$ \\
\# Unigenes & 74,860 \\
N50 & 2,272 \\
Minimum Length & 201 \\
Average Length & 1,098 \\
Maximum Length & 17,667 \\
\hline
\end{tabular}




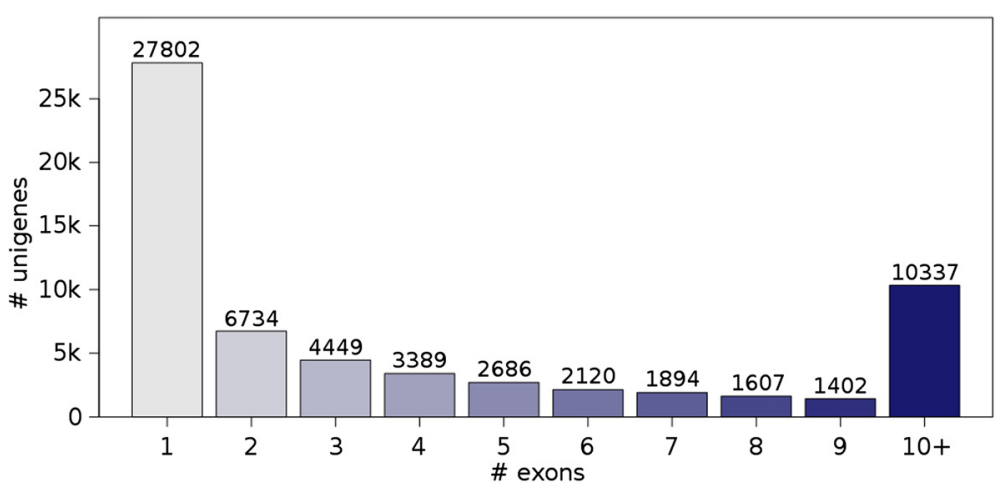

Fig. 1 Distribution of exon counts within the unfiltered assembly. Longer models range from 10 to 119 exons

\section{Annotation with known gene and protein databases} Using blastx, a total of 36,195 unigenes (48 \%) matched to proteins in the non-redundant database (significance defined as an E-value less than 1e-5). To simplify the analysis, only the top hit for each unigene was retained. $35 \%$ of the matches were to equine proteins, and of these, $97 \%$ were computationally derived entries (XP_accession numbers). Additionally, unigenes aligned by BLAT to the equine genome were compared to the NCBI horse RefSeq, NCBI non-horse RefSeq, and Ensembl prediction tracks available from the UCSC Genome Browser. A summary of overlap between the known databases is provided in Table 4.

Gene IDs were assigned to each unigene based on matches to the non-redundant protein database or RefSeq alignments, resulting in annotation of 44,730 transcripts. Unannotated transcripts retained their identifier provided by Trinity. These transcripts likely correspond to novel genes or non-coding RNA and were selected for further examination. This annotated alignment can be loaded into commonly used genome browsers to supplement existing annotation (Fig. 2) [31].

\section{Amplification and sequencing of cDNA from putative novel transcripts}

There were a total of 13,632 unigenes with 3 or more exons that did not match to known RefSeq annotation.

Table 2 Isoform statistics by locus. Unigenes are clustered together into unique loci based on an overlap of at least 1 bp

\begin{tabular}{lll}
\hline Statistics & $\begin{array}{l}\text { All } \\
\text { Transcripts }\end{array}$ & $\begin{array}{l}\text { Long (3+ Exon) } \\
\text { Transcripts }\end{array}$ \\
\hline Total Unigenes & 55,120 & 27,884 \\
Unique Loci & 23,779 & 12,905 \\
Min Unigenes per Locus & 1 & 1 \\
Max Unigenes per Locus & 125 & 89 \\
$\begin{array}{l}\text { Average Unigenes per } \\
\text { Locus }\end{array}$ & 2.32 & 2.16 \\
\hline
\end{tabular}

Of these, there were 4,718 that did not overlap with other unigenes. A subset of 12 unique transcripts that contained ORFs which spanned over 3 exons were selected for molecular validation (Table 5).

RT-PCR successfully amplified cDNA from all selected transcripts. All products were of the expected length and Sanger-derived sequences matched completely with assembled sequences (example in Fig. 3). As differential expression was not the goal of this study, no quantitative analyses were attempted. However, one selected transcript did display a qualitative trend for disease-specific expression (Fig. 4). The best protein match (placenta-specific protein 1 precursor), located on ECAX, is a computational prediction with support from 1 equine mRNA and $85 \%$ coverage of RNA-seq alignments from one sample in the short-read archive. The only other equine-specific protein match was to a homologous gene, placenta-specific protein 1-like $(E=4 e-9)$, which was mapped approximately $100 \mathrm{~kb}$ downstream of the unigene alignment on chromosome 12 . However, this record is completely computationally derived, supported only by similarity to two proteins.

\section{Discussion and conclusion}

We utilized RNA-seq to successfully generate a transcriptome assembly of equine lamellar tissue. As the hoof is a specialized tissue, it likely has unique transcripts that previous annotation efforts would have missed. By pooling data from healthy and diseased tissues, we have captured loci that should be valuable to future differential expression studies. Though the varied physiological states could

Table 3 Mapping statistics for RNA-seq onto the equCab2 reference genome assembly

\begin{tabular}{llllll}
\hline Sample & Phenotype & Total Reads & Mapped Reads & $\%$ Mapped & SNPs \\
\hline CU1 & control & $36,277,643$ & $31,561,549$ & $87 \%$ & 60,580 \\
CU18 & acute & $43,422,463$ & $38,211,767$ & $88 \%$ & 72,281 \\
LSU-J & chronic & $33,278,897$ & $29,618,218$ & $89 \%$ & 58,368 \\
\hline
\end{tabular}


Table 4 Unigenes matching records in selected databases. The repeat-filtered assembly was utilized for EquCab2 alignment-based annotation

\begin{tabular}{lll}
\hline Database & Total Records & Unigenes \\
\hline NCBI NR Protein & $37,818,139$ & $36,195 / 74,860^{\mathrm{a}}$ (48 \%) \\
Equine-Specific Repeats & $2,905,169$ & $19,740 / 74,860^{\mathrm{a}}(26 \%)$ \\
Non-Horse RefSeq & 255,606 & $24,501 / 55,120^{\mathrm{b}}(44 \%)$ \\
Ensembl Predictions & 29,196 & $15,538 / 55,120^{\mathrm{b}}(28 \%)$ \\
Horse RefSeq & 1,169 & $604 / 55,120^{\mathrm{b}}(1 \%)$ \\
None & $\mathrm{n} / \mathrm{a}$ & $31,091 / 74,860^{\mathrm{a}}(42 \%)$ \\
\hline
\end{tabular}

anfiltered transcriptome assembly

${ }^{\mathrm{b}}$ Repeat-filtered transcriptome assembly

result in differences between each transcriptome, pooling the data prior to assembly ensures sufficient power to assemble lower expressed loci. This data set represents a valuable tool for laminitis research, providing information on both known genes expressed in the hoof, as well as a wealth of previously unannotated transcripts. The transcripts identified in this study can now be utilized with other technologies to search for novel targets with relevance to laminitis.

RNA-seq provides unprecedented power for transcript and isoform discovery. However, relatively little of this information trickles down to human-readable annotation and applied datasets useful to the average molecular biologist. While some resources now exist that attempt to bridge this gap by providing bioinformatics instruction for molecular biologists, this approach is not practical for all researchers [32]. Our newly generated data is available in two ways. Raw reads and identified variants have been deposited in public databases, so that they may be accessed or incorporated into automated pipelines. NCBI has recently begun to advantageously incorporate RNA-seq data from the short-read archive into their RefSeq annotation pipeline, and the inclusion of additional unique tissue types is essential for robust annotation from this automated analysis. However, these updated annotations (especially computational predictions) are not always readily accessible in popular genome browsers. Therefore, we have also provided downloadable Browser Extensible Data (BED) tracks of our assembly.

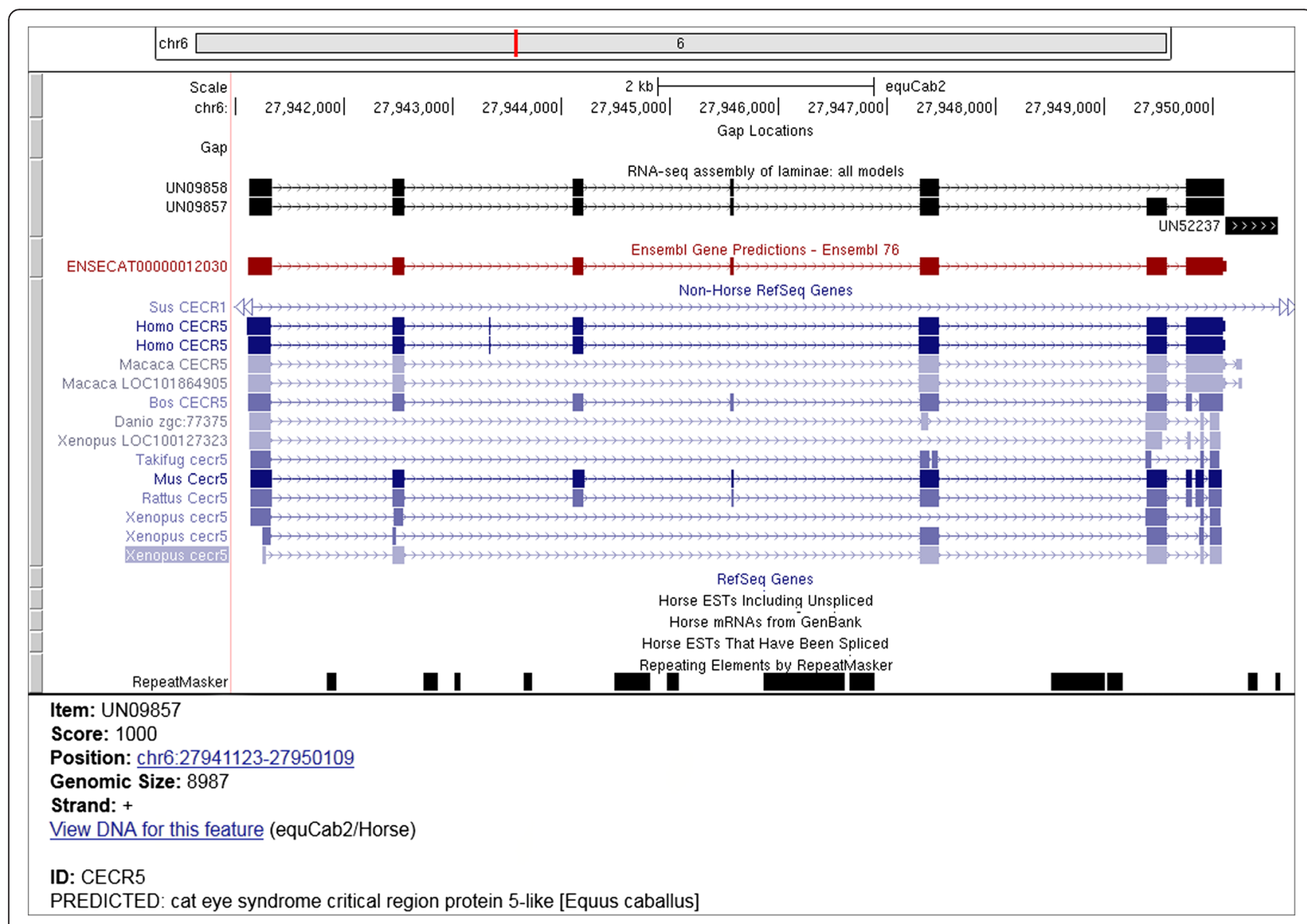

Fig. 2 Example custom annotation on UCSC Genome Browser. Clicking on the identifier "UN09857" loads the screen in the lower panel. Custom identifiers provide corresponding gene and protein annotation for each unigene 
Table 5 Putative novel loci validated by RT-PCR

\begin{tabular}{|c|c|c|c|c|c|c|c|}
\hline Name & Chr & Start & End & $\begin{array}{l}\text { ORF } \\
\text { length }\end{array}$ & Exons & $\mathrm{E}$ & BLASTX \\
\hline UN20159 & 1 & 160767946 & 160771835 & 85 & 4 & $1.2 \mathrm{E}-40$ & AAA80518 T-cell receptor alpha chain (IgC TCRA) [Equus caballus] \\
\hline UN14299 & 4 & 24422341 & 24427035 & 83 & 4 & none & none \\
\hline UN30143 & 5 & 15325547 & 15363449 & 515 & 16 & $7.3 \mathrm{E}-22$ & $\begin{array}{l}\text { XP_003209297 PREDICTED: intraflagellar transport protein } 80 \text { homolog } \\
\text { [Meleagris gallopavo] }\end{array}$ \\
\hline UN27297 & 5 & 74952780 & 74965671 & 177 & 6 & $1.7 \mathrm{E}-56$ & XP_001493637 PREDICTED: guanylate-binding protein 5 [Equus caballus] \\
\hline UN27113 & 9 & 81709438 & 81711119 & 137 & 4 & $2.9 \mathrm{E}-60$ & XP_001917082 PREDICTED: lymphocyte antigen 6H-like [Equus caballus] \\
\hline UN28086 & 11 & 880333 & 894532 & 240 & 6 & $9.9 \mathrm{E}-31$ & $\begin{array}{l}\text { NP_663348 secreted and transmembrane protein 1A precursor [Mus } \\
\text { musculus] }\end{array}$ \\
\hline UN62514 & 11 & 37045433 & 37058857 & 162 & 5 & $1.5 \mathrm{E}-11$ & ACl67873163 Perlwapin [Salmo salar] \\
\hline UN21936 & 12 & 19828520 & 19834360 & 195 & 5 & $2.1 \mathrm{E}-14$ & NP_001243909 placenta-specific protein 1 precursor [Equus caballus] \\
\hline UN70945 & 22 & 34309639 & 34312469 & 141 & 4 & 1.7E-54 & AES10462 antileukoproteinase-like protein [Mustela putorius furo] \\
\hline UN26965 & 24 & 44802220 & 44806274 & 142 & 5 & $3.5 \mathrm{E}-21$ & XP_002696828 PREDICTED: uncharacterized protein LOC509029 [Bos taurus] \\
\hline UN26584 & 28 & 21567866 & 21620053 & 248 & 7 & $5.8 \mathrm{E}-102$ & $\begin{array}{l}\text { XP_003952339 PREDICTED: uncharacterized protein LOC101059192 [Pan } \\
\text { troglodytes] }\end{array}$ \\
\hline UN50658 & $x$ & 2231083 & 2240297 & 174 & 7 & $1.0 \mathrm{E}-38$ & XP_003134963 PREDICTED: odorant-binding protein-like [Sus scrofa] \\
\hline
\end{tabular}

The first file, labeled the "full" assembly, includes models of any number of exons. We have also provided only those models with 3 or more exons (the "larger" assembly) in order to remove partial transcripts likely originating from poorly expressed loci and intronless non-coding RNAs. The BED format is small and much easier to use than the raw sequencing data itself, including only the positions of each feature (not the exact sequence). BED files also are quite easy for individual researchers to load gene model annotation into their browser of choice [33].

Our data also includes potential non-coding RNAs, which are an emerging field of research. As the RefSeq set is specifically designed for protein-coding genes, all other transcript types are not given accession numbers.
There are existing databases of non-coding RNAs available for the human and mouse genomes, however for all other species, there are only the few (less than ten) entries manually curated from the literature [34]. Unlike protein-coding genes, there is considerably less sequence conservation between species in non-coding RNAs, necessitating within species identification [35]. Within non-coding RNAs, there are two main classes: small $(<200 \mathrm{bp})$ and long (>200 bp) [36]. While long noncoding RNAs are often picked up in normal RNA-seq experiments (and must be separated from proteincoding mRNAs for analysis), the smaller molecules are often excluded in normal RNA-seq library preparation, and require additional methodologies to sequence.

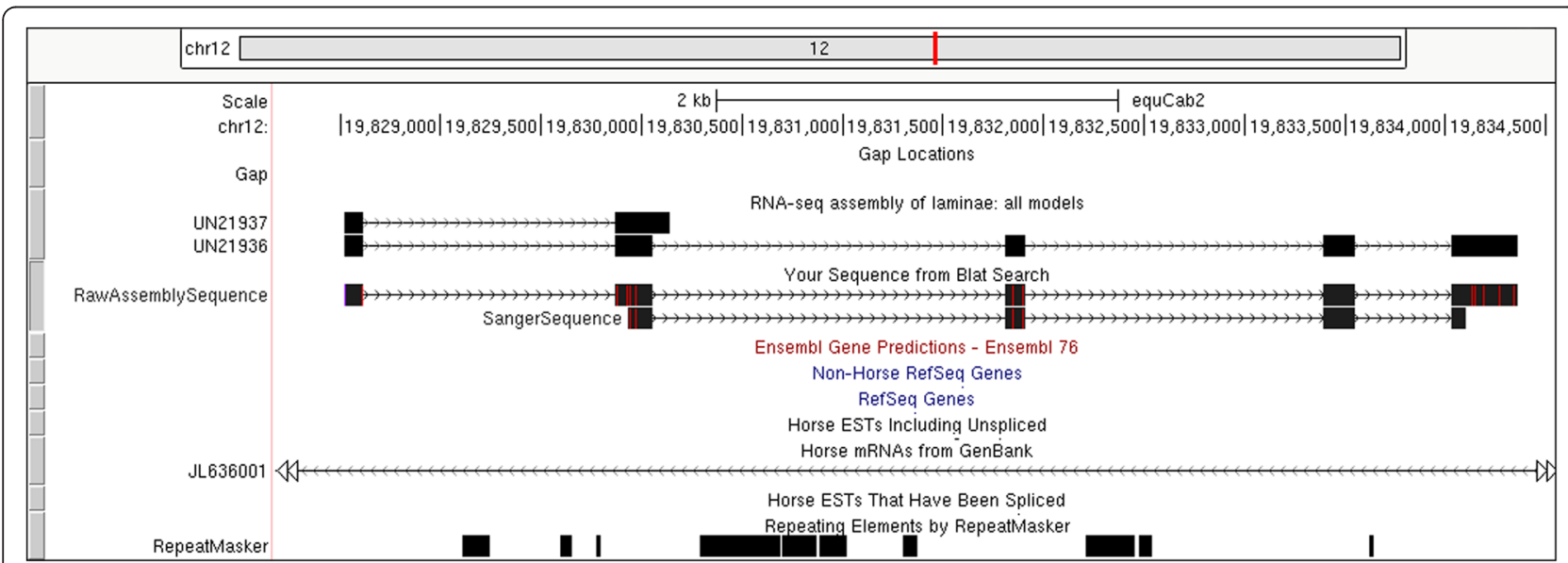

Fig. 3 BLAT alignment of sequenced CDNA from UN21936 and assembled transcripts to the reference genome. Screenshot was captured from the UCSC Genome Browser. Dark boxes represent exons while thin lines are introns. The empty RefSeq Genes, Ensembl Gene Predictions, Horse ESTs, and Non-Horse RefSeq tracks indicate that there has never been expression or computational predictions placed here. Although the amplicon shows three mismatches to the reference (shown as vertical red lines on the SangerSequence data), this sequence aligned perfectly to the sequence from the de novo assembled transcript (RawAssemblySequence) 


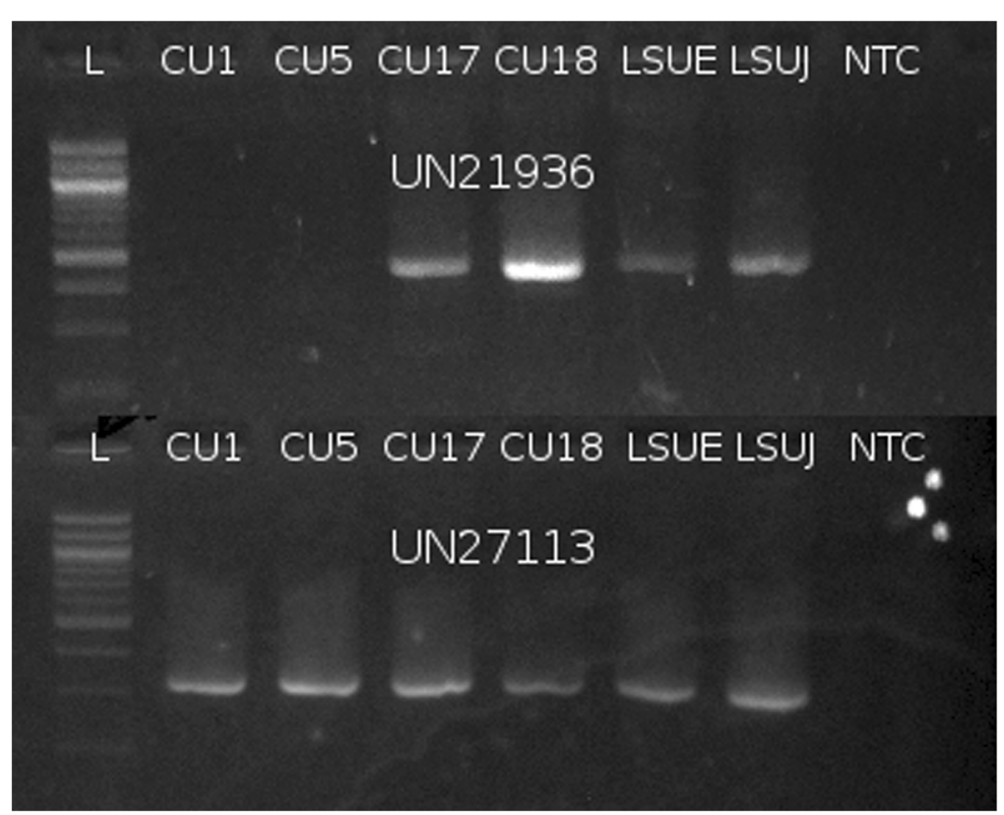

Fig. 4 Agarose gel demonstrating the expression of UN21936 and UN27113. Expression of UN21936 appears to be limited to laminitic samples. CU1, CU5 = control; CU17, CU18 = acute laminitis; LSUE, LSUJ = chronic laminitis; NTC = non-template (negative) control

The function of non-coding RNAs has been the subject of recent controversy. It is debatable whether the observed RNA transcription is biologically relevant, or if transcription may simply be technical noise [37, 38]. Well documented functions for non-coding RNA include regulation of the genome (through chromatin modification, DNA binding, and protein binding) and of cellular differentiation during development [39-41]. One of the most well-known non-coding RNAs is XIST, which regulates $\mathrm{X}$ chromosome inactivation in females. More recently, several mutations that cause overexpression of a conserved long non-coding RNA proved to be responsible for the bovine polled phenotype [42]. It is thus important to consider all possible RNAs in studies of differential expression, instead of only the proteincoding transcripts.

Utilization of this data in studies of laminitis could identify new targets and pathways to help further our understanding of the etiology. Whereas current veterinary methods generally can only detect laminitis at the onset of lameness, the development of biomarkers could allow for rapid identification (and thus the most effective treatment) of cases before permanent damage occurs. Future understanding of the precise pathways underlying laminitis could lead to vital novel prevention methods and treatments.

\section{Methods}

Sample collection and transcriptome sequencing

Samples were collected from four horses presented for necropsy for disposal to the Cornell University College of
Veterinary Medicine (samples labeled CU). An additional two lamellar samples were provided by a collaborator (labeled LSU). Medical history was collected when available. Full-thickness, mid-sagittal hoof sections were placed on ice for transport to the lab, gross examination, and dissection of lamellar tissue. Samples were placed into RNA later (Life Technologies, Carlsbad, CA, USA) and stored at $-80^{\circ} \mathrm{C}$ until processing.

Phenotype was assessed through medical history, physical exam prior to euthanasia, and gross findings. Control animals were defined by the distal phalanx running parallel to the hoof wall, with no bruising or thickening of the laminae. Acute cases often had some degree of rotation and/or sinking, as well as lamellar hemorrhage, edema, and thickening. Chronic cases were defined by thickened, fibrous lamina; variable resorption and/or remodeling of the distal phalanx, often with rotation and/or sinking; and variably severe chronic hemorrhage. Sample information can be found in Table 6 .

RNA was extracted from approximately $60 \mathrm{mg}$ of lamellar tissue using the Qiagen RNeasy kit (Qiagen Inc., Valencia, CA, USA) following manufacturer's protocols for fibrous tissue. $50 \mu \mathrm{L}$ of RNA was DNase treated using either the Ambion Turbo DNA free kit (Life Technologies, Carlsbad, CA, USA) or Qiagen DNase I kit, followed by Qiagen RNA cleanup kit. Quantification was carried out using a NanoDrop spectrophotometer (NanoDrop Technologies LLC., Wilmington, DE, USA).

Library preparation and sequencing was performed by Cornell University's Life Sciences Core Laboratory Center. A total of 5-10 $\mu \mathrm{g}$ of RNA from each sample 
Table 6 Summary of samples used in this study. Laminitis phenotype was determined through medical history and histological examination. Demographic information was not available for most horses

\begin{tabular}{lllllll}
\hline Sample ID & Phenotype & Age & Sex & Breed & History & Experiment \\
\hline CU1 & control & unknown & unknown & unknown & healthy & RNA-seq, RT-PCR \\
CU5 & control & 3 years & gelding & Quarab & healthy & RT-PCR \\
CU17 & acute & 13 years & stallion & unknown & enterocolitis & RT-PCR \\
CU18 & acute & unknown & unknown & unknown & enterocolitis & RNA-seq, RT-PCR \\
LSU-E & chronic & unknown & unknown & unknown & Equine Metabolic Syndrome & RT-PCR \\
LSU-J & chronic & unknown & unknown & unknown & Equine Metabolic Syndrome & RNA-seq, RT-PCR \\
\hline
\end{tabular}

was submitted. Single-end libraries were constructed using manufacturer's protocols for poly-T selection and sequenced on an Illumina HiSeq 2000 (Illumina Inc., San Diego, CA, USA). Raw reads were submitted to the European Nucleotide Archive [ENA:PRJEB6100].

\section{De novo assembly}

Raw RNA-seq reads were processed in two steps. First, a custom $\mathrm{R}$ script (based on the ShortRead package) was used to remove adapter and barcode sequences, as well as to trim low quality $(\mathrm{Q}<20)$ bases from both ends of the reads [43]. Trimmed reads shorter than 25 bp were discarded. Second, reads were aligned to the GenBank virus (version 186) and ribosomal RNA sequence databases with BWA under default parameters [44]. Only unmapped reads were retained for assembly.

The filtered reads from all samples were pooled and de novo assembled into contigs using Trinity with "min_kmer_cov" set to 2 [45]. In order to remove some of the redundancy of Trinity-generated contigs, a further assembly step using iAssembler with a minimum of $99 \%$ identity (-p) was performed [25]. Contigs shorter than 200 bp were discarded.

\section{Unigene annotation}

All unique transcripts (unigenes) were compared to the GenBank non-redundant protein database using blastx with an E-value cutoff of 1e-5. Only the protein with the lowest E-value (and thus highest significance) was retained for further analysis.

Unigenes were also aligned to the equCab 2.0 reference genome using BLAT with parameters recommended for same-species mRNA alignments [46]. The pslCDnaFilter tool was used to remove alignments with less than $200 \mathrm{bp}$, $98 \%$ identity, or $50 \%$ coverage. The resulting PSL file was converted to BED format and compared with Equinespecific repeat annotation using BEDtools intersectBed in order to filter out alignments that contained over $10 \%$ repetitive DNA [47, 48]. Many retroviruses in the genome are expressed, but high homology among these elements often leads to chimeric and spurious assemblies, and thus creates problems for alignment-based analyses. The filtered unigenes were then compared to NCBI Non-Horse RefSeq, Horse RefSeq, and Horse Ensembl annotations using intersectBed at $10 \%$ overlap.

Putative gene names were assigned to unigenes based on high quality matches to NCBI non-redundant databases. Two BED files were produced for use in genome

Table 7 Primers used to confirm expression of unannotated transcripts. All PCRs were performed with an annealing temperature of $62^{\circ} \mathrm{C}$ and an elongation time of $30 \mathrm{~s}$

\begin{tabular}{|c|c|c|c|}
\hline Name & Forward Seq & Reverse Seq & Size \\
\hline UN20159 & TTCAAGAGCAATGGGATGCT & CGCAGTGTCATGAACAGGTTA & $227 \mathrm{bp}$ \\
\hline UN14299 & TITTCCTCTGAAGCATTTCC & TAGAGCATCGCTTTCCTGGT & $284 \mathrm{bp}$ \\
\hline UN30143 & CCCACCCCCAACCTAGATAC & AGGTAAGACAGGCTGGGTCA & $499 \mathrm{bp}$ \\
\hline UN27297 & GTCCGAATTCAGCCAATCAT & GAAACGATTTATGGCCTCCA & $495 \mathrm{bp}$ \\
\hline UN27113 & TGAAAGGCATCCATCTGGTC & ACCCCGTTACAGAGGTCCTT & $329 \mathrm{bp}$ \\
\hline UN28086 & TCCTTGCTAGGATGCTCTGG & GAGCACCAGGATGAAGAGGA & $506 \mathrm{bp}$ \\
\hline UN62514 & GGCTCCTCCTCCTTGTGAG & AACAGCAGTTTGGCAGGAGT & $437 \mathrm{bp}$ \\
\hline UN21936 & CTATGTTCTGGGCTGTGGTG & TGTAGCCACGTTTGCACTCT & $485 \mathrm{bp}$ \\
\hline UN70945 & CCTCATGACCTTCGTGGTTC & ATCTITITGAGCTGGCAAGG & $409 \mathrm{bp}$ \\
\hline UN26965 & GCACCCTACTCCCACATACG & GCTCACATCCACGTCTGCTA & $422 \mathrm{bp}$ \\
\hline UN26584 & GTACATTCCTCCCCTGCAAA & TCGACACCATCCAGTTGAAA & $479 \mathrm{bp}$ \\
\hline UN50658 & CTGACCAGGACCCTCAGTCT & TCAGTGACCAGGCCTTCTTC & $343 \mathrm{bp}$ \\
\hline
\end{tabular}


browsers (one containing all transcripts and one with only large transcripts containing 3 or more exons) [31].

\section{Variant calling}

Raw sequencing reads were split by barcode and aligned to the EquCab 2.0 reference genome using BWA under default parameters. SAMtools was used to convert alignments to BAM format and to remove PCR duplicate reads [49]. SNPs were identified with GATK using the recommended pipelines with a $\mathrm{Q}>30$ cutoff [28-30]. VCFtools was then used to filter out variants with fewer than 10 observations, followed by BEDtools to remove variants that fell outside of regions with corresponding assembly alignments [50]. The final list of variants was pooled and submitted to NCBI dbSNP.

\section{Analysis of putative novel loci}

We screened the transcriptome assembly for novel loci with two steps. First, a second genome alignment was prepared by running RepeatMasker (using RepBase 201304-22 libraries) on the unigenes, then BLAT and subsequent filtering was performed as before [51, 52]. Next, the unmasked and masked alignments were compared, and unigenes that passed filtering criteria in both datasets were selected. The unmasked alignments of these unigenes were then compared to RefSeq annotation using BEDtools, and alignments with less than $5 \%$ overlap to known annotation were labeled as putative novel loci. All matches to the unassembled chromosome (chrUn) were discarded. Although valuable novel genes are likely to be found there, the incomplete state of assembly in this region makes downstream alignment based analyses problematic.

Twelve novel genes were selected for RT-PCR validation and proof of concept based on additional criteria. ExPasy "translate" tool was used to identify open reading frames (ORFs) in these unigenes [53]. These were then aligned back to the equCab 2.0 reference genome using BLAT, and only unigenes with ORFs spanning at least three exons on their corresponding transcript annotation were retained, thus identifying larger transcripts with significant exon/intron structure. The ORFs were then compared to the non-redundant protein database using blastp, and targets with little to no experimental data were selected for further validation.

Within each gene, an amplicon of cDNA was targeted using intron spanning primers created with the Primer3 software (Table 7) [54]. Two-step RT-PCR was performed in $15 \mu \mathrm{L}$ reactions with $1 \mu \mathrm{g}$ RNA using the SuperScript VILO MasterMix kit (LifeTechnologies, Carlsbad, CA, USA) followed by standard PCR. $1 \mu \mathrm{L}$ of cDNA was amplified in $10 \mu \mathrm{L}$ PCR with FastStart Taq DNA polymerase (Roche Applied Science, Branford, CT, USA) and included all reagents per the manufacturers recommended conditions.
Amplification was verified on $3 \%$ agarose gel, and the resulting PCR products were submitted to the Cornell Core Life Sciences Laboratories Center for sequencing using standard ABI chemistry on a 3730 DNA Analyzer (Applied Biosystems Inc., Foster City, CA, USA). Amplicons were aligned to their corresponding unigenes to confirm identity using Consed [55].

\section{Availability of supporting data}

The data sets supporting the results of this article are available in the NAGRP Host of Supplementary Data to Publications repository, http://www.animalgenome.org/ repository/pub/CORNEL2015.0126/.

\section{Competing interests}

The authors declare that they have no competing interests.

\section{Authors' contributions}

$\mathrm{HMH}, \mathrm{SG}, \mathrm{ZF}$, and SAB designed experiments. CA and HMH carried out molecular work. HMH, SG and ZF performed bioinformatics analyses. HMH and SAB prepared the manuscript. All authors read and approved the final manuscript.

\section{Authors' information}

Not applicable

\section{Availability of data and materials}

Not applicable

\section{Acknowledgements}

The authors would like to thank Dr. Laura Riggs and the Cornell University Equine Hospital for the samples used in this study. They would also like to thank Dr. Hannah Galantino-Homer for her discussions on disease pathology and Dr. David Adelson for his computational insight as well as for providing more comprehensive equine repeat annotation.

\section{Author details}

'Department of Animal Sciences, University of Florida, Gainesville, FL 32611 USA. ${ }^{2}$ Boyce Thompson Institute for Plant Research, Cornell University, Ithaca, NY 14853, USA. ${ }^{3}$ Laboratory of Molecular Immunoregulation, National Cancer Institute, Bethesda, MD 20892, USA.

Received: 30 January 2015 Accepted: 22 September 2015

Published online: 03 October 2015

\section{References}

1. Pollitt CC. The anatomy and physiology of the suspensory apparatus of the distal phalanx. Vet Clin North Am Equine Pract. 2010;26(1):29-49.

2. Eades SC. Overview of current laminitis research. Vet Clin North Am Equine Pract. 2010;26(1):51-63.

3. van Eps AW, Pollitt CC, Underwood C, Medina-Torres CE, Goodwin WA Belknap JK. Continuous digital hypothermia initiated after the onset of lameness prevents lamellar failure in the oligofructose laminitis model. Equine Vet J. 2014;46(5):625-30.

4. Service USAaPHI, System NAHM. National Economic Cost of Equine Lameness, Colic, and Equine Protozoal Myeloencephalitis (EPM) in the United States. Fort Collins: U.S. Department of Agriculture, APHIS; 2001.

5. Moore RM, Bud, Jenne M. "Vision 20/20 - Conquer Laminitis by 2020". J Equine Veterinary Sci. 2010;30(2):74-76.

6. Faleiros RR, Johnson PJ, Nuovo GJ, Messer NT, Black SJ, Belknap JK. Laminar leukocyte accumulation in horses with carbohydrate overload-induced laminitis. J Vet Intern Med. 2011;25(1):107-15.

7. Leise BS, Faleiros RR, Watts M, Johnson PJ, Black SJ, Belknap JK. Laminar inflammatory gene expression in the carbohydrate overload model of equine laminitis. Equine Vet J. 2011;43(1):54-61.

8. Kwon S, Moore JN, Robertson TP, Hurley DJ, Wagner B, Vandenplas ML. Disparate effects of LPS infusion and carbohydrate overload on 
inflammatory gene expression in equine laminae. Vet Immunol Immunopathol. 2013;155(1-2):1-8.

9. Steelman SM, Johnson D, Wagner B, Stokes A, Chowdhary BP. Cellular and humoral immunity in chronic equine laminitis. Vet Immunol Immunopathol. 2013;153(3-4):217-26.

10. Wang L, Pawlak EA, Johnson PJ, Belknap JK, Eades S, Stack S, et al. Impact of laminitis on the canonical Wnt signaling pathway in basal epithelial cells of the equine digital laminae. PLoS One. 2013;8(2):e56025.

11. Wang L, Pawlak EA, Johnson PJ, Belknap JK, Alfandari D, Black SJ. Expression and activity of collagenases in the digital laminae of horses with carbohydrate overload-induced acute laminitis. J Vet Intern Med. 2014;28(1):215-22.

12. Budak MT, Orsini JA, Pollitt CC, Rubinstein NA. Gene expression in the lamellar dermis-epidermis during the developmental phase of carbohydrate overload-induced laminitis in the horse. Vet Immunol Immunopathol. 2009;131(1-2):86-96.

13. Noschka E, Vandenplas ML, Hurley DJ, Moore JN. Temporal aspects of laminar gene expression during the developmental stages of equine laminitis. Vet Immunol Immunopathol. 2009;129(3-4):242-53.

14. Coleman SJ, Zeng Z, Wang K, Luo S, Khrebtukova I, Mienaltowski MJ, et al. Structural annotation of equine protein-coding genes determined by mRNA sequencing. Anim Genet. 2010;41 Suppl 2:121-30.

15. Coleman SJ, Zeng Z, Hestand MS, Liu J, Macleod JN. Analysis of unannotated equine transcripts identified by mRNA sequencing. PLoS One. 2013;8(7):e70125.

16. 't Hoen PA, Ariyurek Y, Thygesen HH, Vreugdenhil E, Vossen RH, de Menezes $R X$, et al. Deep sequencing-based expression analysis shows major advances in robustness, resolution and inter-lab portability over five microarray platforms. Nucleic Acids Res. 2008;36(21):e141.

17. Malone $\mathrm{JH}$, Oliver B. Microarrays, deep sequencing and the true measure of the transcriptome. BMC Biol. 2011;9:34.

18. Wilson LO, Spriggs A, Taylor JM, Fahrer AM. A novel splicing outcome reveals more than 2000 new mammalian protein isoforms. Bioinformatics. 2014;30(2):151-6.

19. Marioni JC, Mason CE, Mane SM, Stephens M, Gilad Y. RNA-seq: an assessment of technical reproducibility and comparison with gene expression arrays. Genome Res. 2008;18(9):1509-17.

20. Mooney M, Bond J, Monks N, Eugster E, Cherba D, Berlinski P, et al. Comparative RNA-Seq and microarray analysis of gene expression changes in B-cell lymphomas of Canis familiaris. PLoS One. 2013;8(4):e61088.

21. Zhao S, Fung-Leung WP, Bittner A, Ngo K, Liu X. Comparison of RNA-Seq and microarray in transcriptome profiling of activated T cells. PLOS One. 2014;9(1), e78644.

22. Park KD, Park J, Ko J, Kim BC, Kim HS, Ahn K, et al. Whole transcriptome analyses of six thoroughbred horses before and after exercise using RNASeq. BMC Genomics. 2012;13:473.

23. Capomaccio S, Vitulo N, Verini-Supplizi A, Barcaccia G, Albiero A, D'Angelo $M$, et al. RNA sequencing of the exercise transcriptome in equine athletes. PLoS One. 2013;8(12):e83504.

24. Iqbal K, Chitwood JL, Meyers-Brown GA, Roser JF, Ross PJ. RNA-seq transcriptome profiling of equine inner cell mass and trophectoderm. Biol Reprod. 2014;90(3):61.

25. Zheng $Y$, Zhao L, Gao J, Fei Z. iAssembler: a package for de novo assembly of Roche-454/Sanger transcriptome sequences. BMC Bioinformatics. 2011;12:453.

26. NAGRP Host of Supplementary Data to Publications. [http:// www.animalgenome.org/repository/pub/CORNEL2015.0126/]

27. Wade CM, Giulotto E, Sigurdsson S, Zoli M, Gnerre S, Imsland F, et al. Genome sequence, comparative analysis, and population genetics of the domestic horse. Science. 2009;326(5954):865-7.

28. McKenna A, Hanna M, Banks E, Sivachenko A, Cibulskis K, Kernytsky A, et al The Genome Analysis Toolkit: a MapReduce framework for analyzing next-generation DNA sequencing data. Genome Res. 2010;20(9):1297-303.

29. DePristo MA, Banks E, Poplin R, Garimella KV, Maguire JR, Hartl C, et al. A framework for variation discovery and genotyping using next-generation DNA sequencing data. Nat Genet. 2011;43(5):491-8.

30. Van der Auwera GA, Carneiro MO, Hartl C, Poplin R, Del Angel G, Levy-Moonshine A, et al. From FastQ data to high confidence variant calls: the Genome Analysis Toolkit best practices pipeline. Curr Protoc Bioinformatics. 2013;11(1110):11.10.11-33.
31. Horse Genome Analysis Data Repository. [http://www.animalgenome.org/ repository/horse/]

32. Bradnam K, Korf I. UNIX and Perl to the Rescue!: A Field Guide for the Life Sciences (and Other Data-rich Pursuits). Cambridge: Cambridge University Press; 2012.

33. Dreszer TR, Karolchik D, Zweig AS, Hinrichs AS, Raney BJ, Kuhn RM, et al. The UCSC Genome Browser database: extensions and updates 2011. Nucleic Acids Res. 2012:40(Database issue):D918-923.

34. Amaral PP, Clark MB, Gascoigne DK, Dinger ME, Mattick JS. IncRNAdb: a reference database for long noncoding RNAs. Nucleic Acids Res. 2011;39(Database issue):D146-151.

35. Qu Z, Adelson DL. Identification and comparative analysis of ncRNAs in human, mouse and zebrafish indicate a conserved role in regulation of genes expressed in brain. PLoS One. 2012;7(12):e52275.

36. Veneziano D, Nigita G, Ferro A. Computational Approaches for the Analysis of ncRNA through Deep Sequencing Techniques. Front Bioeng Biotechnol. 2015:3:77.

37. van Bakel H, Nislow C, Blencowe BJ, Hughes TR. Most "dark matter" transcripts are associated with known genes. PLoS Biol. 2010;8(5):e1000371.

38. Kapranov P, St Laurent G. Dark Matter RNA: Existence, Function, and Controversy. Front Genet. 2012;3:60.

39. Rinn JL, Chang HY. Genome regulation by long noncoding RNAs. Annu Rev Biochem. 2012;81:145-66.

40. Hu W, Alvarez-Dominguez JR, Lodish HF. Regulation of mammalian cell differentiation by long non-coding RNAs. EMBO Rep. 2012;13(11):971-83.

41. Morán I, Akerman I, van de Bunt M, Xie R, Benazra M, Nammo T, et al. Human $\beta$ cell transcriptome analysis uncovers IncRNAs that are tissuespecific, dynamically regulated, and abnormally expressed in type 2 diabetes. Cell Metab. 2012;16(4):435-48.

42. Allais-Bonnet A, Grohs C, Medugorac I, Krebs S, Djari A, Graf A, et al. Novel insights into the bovine polled phenotype and horn ontogenesis in Bovidae. PLoS One. 2013;8(5):e63512.

43. Morgan $M$, Anders $S$, Lawrence $M$, Aboyoun $P$, Pagès $H$, Gentleman $R$. ShortRead: a bioconductor package for input, quality assessment and exploration of high-throughput sequence data. Bioinformatics. 2009;25(19):2607-8.

44. Li H, Durbin R. Fast and accurate long-read alignment with BurrowsWheeler transform. Bioinformatics. 2010;26(5):589-95.

45. Grabherr MG, Haas BJ, Yassour M, Levin JZ, Thompson DA, Amit I, et al. Full-length transcriptome assembly from RNA-Seq data without a reference genome. Nat Biotechnol. 2011;29(7):644-52.

46. Kent WJ. BLAT-the BLAST-like alignment tool. Genome Res. 2002;12(4):656-64.

47. Pruitt KD, Brown GR, Hiatt SM, Thibaud-Nissen F, Astashyn A, Ermolaeva O, et al. RefSeq: an update on mammalian reference sequences. Nucleic Acids Res. 2014:42(Database issue):D756-763.

48. Quinlan AR, Hall IM. BEDTools: a flexible suite of utilities for comparing genomic features. Bioinformatics. 2010;26(6):841-2

49. Li H, Handsaker B, Wysoker A, Fennell T, Ruan J, Homer N, et al. The Sequence Alignment/Map format and SAMtools. Bioinformatics. 2009;25(16):2078-9.

50. Danecek P, Auton A, Abecasis G, Albers CA, Banks E, DePristo MA, et al. The variant call format and VCFtools. Bioinformatics. 2011;27(15):2156-8.

51. Smit A, Hubley R, Green P. RepeatMasker Open-3.0. In: 1996-2010: http:// www.repeatmasker.org/.

52. Jurka J, Kapitonov W, Pavlicek A, Klonowski P, Kohany O, Walichiewicz J. Repbase Update, a database of eukaryotic repetitive elements. Cytogenet Genome Res. 2005;110(1-4):462-7.

53. Gasteiger E, Gattiker A, Hoogland C, Ivanyi I, Appel RD, Bairoch A. ExPASy: the proteomics server for in-depth protein knowledge and analysis. Nucleic Acids Res. 2003;31(13):3784-8.

54. Rozen S, Skaletsky H. Primer3 on the WWW for general users and for biologist programmers. Methods Mol Biol. 2000;132:365-86.

55. Gordon D, Abajian C, Green P. Consed: a graphical tool for sequence finishing. Genome Res. 1998:8(3):195-202. 